\title{
LA INFORMACIÓN EN LA ERA DE INTERNET. EL CASO DE LAS FAKE NEWS
}

\section{MiguEL J. ARJONA SÁNCHEZ ${ }^{1}$}

RESUMEN: El fenómeno de las fake news ha puesto sobre la mesa el problema de la desinformación. Un problema enfrentado de forma muy distinta en los Estados Unidos y en Europa; el primero a favor de una muy liberal concepción del mercado de las ideas, y el segundo con una concepción más limitadora del derecho de expresión e información. Pero detrás de las fake news se esconden también las dificultades de enfrentar un nuevo espacio que no es otro que el ciberespacio, donde la información se mueve en flujos difícil de aprehender. Un nuevo espacio que nos interroga sobre si está legitimado un cambio en el paradigma constitucional, especialmente si se considera que está generando una nueva elite social (la netocracia) que reclama un nuevo tratamiento jurídico (the code).

PalabRas Clave: Fake News; derecho de expresión; derecho de información; ciberespacio; netocracia.

ABSTRACT: The phenomenon of fake news has put the problem of misinformation on the table. A problem faced very differently in the United States and in Europe; the first in favor of a very liberal conception of the market of ideas, and the second with a more limiting conception of the right to expression and information. But behind the fake news there are also the difficulties of facing a new space that is none other than cyberspace, where information moves in flows that are difficult to grasp. A new space that questions us about whether a change in the constitutional paradigm is legitimized, especially if it is considered to be generating a new social elite (the netocracy), that demands a new legal treatment (the code).

Keywords: Fake news; right of expression; right of information; cyberspace; netocracy.

1 Profesor de Derecho Constitucional Universidad de Granada (España). Email: miguelarjona@ugr.es. 


\section{LAS FAKE NEWS Y LA DESINFORMACIÓN}

\subsection{Las "noticias falsas"}

El diccionario de Cambridge definió a las fake news como "historias falsas que parecen ser noticias, difundidas en Internet o utilizando otros medios, generalmente creadas para influir en opiniones políticas o como una broma" ${ }^{2}$. El término, cuyo uso se ha generalizado incluso en los países de habla no inglesa, ha sido criticado por la doctrina por su falta de precisión y su insuficiencia para describir y definir un fenómeno que comprendería un gran número de actividades muy diversas en su modus operandi.

Sin embargo, el uso de historias falsas con la apariencia de noticias no es nuevo y ha venido sucediéndose desde la aparición de los medios de comunicación de masas con la generalización de la prensa. A finales del siglo XIX ya es reconocible el uso de las noticias falsas en el denominado periodismo amarillo (o yellow journalism), como el caso de los tabloides del magnate estadounidense de la prensa Randolph Hearst (inmortalizado por Orson Welles en su genial Citizen Kane ${ }^{3}$ ) responsable de la campaña de propaganda que dio cobertura a la intervención estadounidense en Cuba y la consecuente guerra hispanoamericana.

Pese a su popularidad, el término no ha satisfecho a todos; el informe del Grupo Independiente de Alto nivel sobre las Fake News y la información on line (HLEG) creado en 2018 por la Comisión Europea ${ }^{4}$ (para el asesoramiento de aquellas políticas que pudieran contrarrestar este fenómeno, y que, paradójicamente, fue intitulado bajo la denominación de fake news) recomendó el más apropiado uso del término "desinformación", que englobaría a "todas las formas de información falsa, inexacta o engañosa diseñada, presentada y proporcionada para causar daño o beneficio público intencionadamente ${ }^{\prime \prime}$. El reporte excluía expresamente a aquellas otras formas deliberadas de distorsión de la información, que como la sátira o la parodia $^{6}$ no son engañosas, y cuyo combate representaría un gran retroceso para la libertad de expresión.

\footnotetext{
2 "False stories that appear to be news, spread on the internet or using other media, usually created to influence political views or as a joke". Cambridge Dictionary, disponible en Web: https://dictionary.cambridge.org/dictionary/english/fake-news

${ }^{3}$ WELLES, O. (productor) y WELLES, O. (director). (1941). Citizen Kane [cinta cinematográfica]. Estados Unidos: Mercury Productions.

4 Véase: EUROPEAN COMMISSION. DIRECTORATE-GENERAL FOR COMMUNICATION NETWORKS, CONTENT AND TECHNOLOGY. "A multi-dimensional approach to disinformation: Report of the independent high-level group on fake news and online disinformation", Publications Office of the European Union, 2018, p. 3. Disponible en Internet: https://ec.europa.eu/digital-single-market/en/news/final-report-high-level-expert-group-fakenews-and-online-disinformation

5 "In this Report, we favour the word "disinformation" over "fake news". Disinformation, as used in the Report, includes all forms of false, inaccurate, or misleading information designed, presented and promoted to intentionally cause public harm or for profit". Ibíd., 3.

${ }^{6}$ Ibíd.
} 
La mera propuesta definitoria del HLEG, que exige el respeto a ciertos parámetros de la calidad de las informaciones (no debe ser "falsa, inexacta o engañosa" y no debe estar "diseñada, presentada y proporcionada para causar daño o beneficio público intencionadamente") nos conduce a la pregunta sobre las exigencias de proporcionar una información veraz. Una cuestión, sin embargo, en nada pacífica.

\subsection{La desinformación en los medios}

La función propagandística de los medios de comunicación de masas fue puesta de manifiesto en 1988 por Noam Chomsky y Edward S. Herman en su influyente "Manufacturing consent: The political economy of the mass media"7. Dicha obra, de enorme impacto y calado pese a su sencillez, describe a los mass media estadounidenses (sus conclusiones son ampliables) como unas eficaces empresas ideológicas que despliegan una verdadera función propagandística, gracias a su posición preponderante en el mercado del entretenimiento. A diferencia de lo que ocurre en los regímenes totalitarios, que a menudo recurren a la coerción manifiesta a la prensa, en las sociedades democráticas será su propia autocensura la que desvirtuará su original función informativa. Mediante la difusión masiva de mensajes sesgados, previamente confeccionados a partir de las suposiciones previas internalizadas por la población, los medios serán capaces de generar el debido "consent" 8 que asegure el mantenimiento del status quo. El mero peso cuantitativo de estas informaciones silenciará a aquella contracorriente, que deberán enfrentarse a la ardua labor de hacerse escuchar y entender frente a las voces amplificadas de los grandes medios, confrontando simultáneamente los prejuicios y clichés ya asumidos como consensos por el público. Los grandes medios servirán al poder informando sesgadamente, o sea, desinformando.

Las demoledoras conclusiones de Chomsky y Herman objetaron la pretendida imparcialidad de los medios, al evidenciar su papel en la trama del poder. En las propias palabras de los autores, la prensa "ajusta las premisas del discurso dominante" y "decide lo que la población en general puede ver, escuchar y pensar gestionando la opinión pública a través de la propaganda" ${ }^{\prime 9}$. Como sus autores nos

7 HERMAN, E. S., \& CHOMSKY, N., Manufacturing consent: The political economy of the mass media, Random House, 2010.

${ }^{8}$ Los autores tomaron prestada la expresión "manufacturing the consens" ya empleada por Walter Lippmann en su libro Public Opinion de 1922, y a la sazón galardonado con el Premio Pulitzer. Véase: LIPPMANN, W., Public Opinion. 1922. URL: http://infomotions. com/etexts/gutenberg/dirs/etext04/pbp nn10. htm.

9 "Perhaps this is an obvious point, but the democratic postulate is that the media are independent and committed to discovering and reporting the truth, and that they do not merely reflect the world as powerful groups wish it to be perceived. Leaders of the media claim that their news choices rest on unbiased professional and objective criteria, and they have support for this contention in the intellectual community. If however, the powerful are able to fix the premises of discourse, to decide what the general populace is allowed to see, hear, and think about, and to 
recordarán, los grandes medios no dejan de ser grandes conglomerados empresariales controlados por otras corporaciones o individuos muy ricos ${ }^{10}$. Una visión, nada complaciente en términos democráticos con el papel de la denominada prensa del mundo libre, que sería completada con otra original aportación, la de las Diez estrategias de manipulación mediática, que el profesor Chomsky daría a conocer en el año 2010, y en el que concretaría las principales técnicas propagandísticas de los medios ${ }^{11}$.

\subsection{Desinformación y fake news}

Desconocemos de si conscientes de esta crítica, lo cierto es que el Grupo Independiente de Alto nivel sobre las Fake News y la información on line precisó que prefería evitar el término "noticias falsas" para precisar de forma más precisa el tipo de actividades a enfrentar. Apoyándose en un informe de tan sólo un año anterior (del 2017) del Consejo de Europa denominado "Information disorder: Toward an interdisciplinary framework for research and policy making" de Claire Wardle y Hossein Derakhshan $^{12}$, el HLEG advirtió de la dificultad de caracterizar al contenido de estas denominadas "noticias" como completamente falso, para optar por caracterizarlo como una "información fabricada mezclada con hechos y prácticas que van mucho más allá de cualquier cosa que se parezca a "noticias"”'. El informe incluyó toda una lista de actividades entre las que se encuentra el astroturfing, las redes de trolling, los miembros visuales, etc y de actividades aún mucho más difícil de calificar como la mera publicación, el comentario, twittear o retuitear, $\ldots{ }^{13}$.

\section{El tratamiento constitucional de la información}

E La acusación realizada sobre la actividad propagandística de los medios, que sería muy contestada, supondría todo un reto para la teoría liberal de los medios de comunicación, sobre la que se sigue basando gran parte de su regulación en

\footnotetext{
"manage" public opinion by regular propaganda campaigns, the standard view of how the system works is at serious odds with reality". HERMAN, E. S., \& CHOMSKY, N., Op. Cit. p. XI (Preface). ${ }^{10}$ Ibíd., p. 332.

${ }^{11}$ Estas estrategias son: "La estrategia de la distracción, "crear problemas para después ofrecer soluciones", "la estrategia de la gradualidad", "la estrategia de diferir", "dirigirse al público como criaturas de poca edad", "utilizar el aspecto emocional mucho más que la reflexión", "mantener al público en la ignorancia y la mediocridad", "estimular al público para ser complaciente con la mediocridad", "reforzar la autoculpabilidad" y "conocer a los individuos mejor que lo que ellos se conocen". CHOMSKY, N., 10 strategies of manipulation» by the media, 2011. URL: http://parisis. files. wordpress. com/2011/01/noam-chomsky.pdf

12 WARDLE, C Y DERAKHSHAN, H "Information disorder: Toward an interdisciplinary framework for research and policy making", Council of Europe, Strasbourg, 2017.

13 EUROPEAN COMMISSION. DIRECTORATE-GENERAL FOR COMMUNICATION NETWORKS, "Content and Technology. A multi-dimensional approach to disinformation: Report of the independent high-level group on fake news and online disinformation", Op. Cit., 10.
} 
relación con la democracia. Conformada mediante una simple traslación del librecambismo económico al mundo de las ideas, la teoría liberal de los medios gira en torno a la consideración de la competencia en el mercado (de las ideas) como la mejor forma de acceso a la verdad ${ }^{14}$. La prensa asegura que el gran público tenga acceso a las ideas, mientras que su papel de monitorización del poder asegura la participación de las masas desposeídas de la propiedad en su autogobierno, lo que le confiere al gobierno democrático su fortaleza, lo que asegura las libertades individuales ${ }^{15}$. Como resulta obvio, ello necesita de un mercado libre donde las iniciativas periodísticas tengan cabida y puedan provocar el eco que merezcan sus contenidos.

\subsection{El paradigma liberal en los Estados Unidos}

El mejor ejemplo de la traslación jurídica de la teoría liberal de los medios de comunicación lo encontramos en el constitucionalismo estadounidense, y concretamente en el desarrollo jurisprudencial de su Primera Enmienda. Desprovista inicialmente de una Bill of Rights, la Constitución estadounidense incorpora su Carta de derechos a través de sus primeras diez enmiendas. La primera prohibirá al Congreso cualquier legislación que adopte a una religión como oficial del Estado o que coarte la libertad de palabra o imprenta o el derecho del pueblo para reunirse pacíficamente y para pedir al gobierno la reparación de agravios. Sobre esta enmienda y su reconocimiento del derecho a la libertad de prensa, la jurisprudencia irá construyendo un enfoque liberal en base a muy pocas distinciones, como aquella que establece cuando nos encontramos ante asuntos y personajes públicos o privados y entre hechos $u$ opiniones, siendo las opiniones completamente libres, puesto que su "enjuiciamiento" queda a merced del mercado ${ }^{16}$.

Con la traslación de las ideas librecambistas al "mercado de las ideas", también se incorporará la fe en "la mano oculta" del mercado, que debiera conducir al consumidor a elegir inteligentemente la mejor idea y rechazar aquellas opiniones que son desechables. En consecuencia, la mejor política pública no podrá ser otra que la ausencia regulatoria, es decir, la plasmación en el mundo de la información del "laissez faire, laissez passer" 17 .

${ }^{14}$ Véase: RODRÍGUEZ-POLO, X. R., \& ALGARRA, M. M., Los principios del sistema liberal de medios ante la crisis de la comunicación pública. Una aproximación crítica. Vivat Academia, 2011, (117), 77-90, 82.

${ }^{15}$ Así se explica la función de monitoreo del poder para la teoría libera. ROBERT W. MCCHESNEY, "Liberalism and the media. Open Democracy blog", 2014. URL: https://www.opendemocracy.net/en/liberalism-and-media/

16 Véase: KANS, M., Freedom of Speech, Yale Law School, 2009.

17 Véase: RODRÍGUEZ-POLO, X. R., \& ALGARRA, M. M. Los principios del sistema liberal de medios ante la crisis de la comunicación pública. Una aproximación crítica, Op. Cit., 81. 
Esta perspectiva será expresada por el juez Holmes de forma excepcionalmente clara, a través de uno de sus votos particulares en la famosa sentencia del caso Abrams v. United States de 1919, en el que señalará que el "best test of truth is the power of the thought to get itself accepted in the competition of the market"18. Pese al gran éxito cosechado por este voto particular, tanto entre la opinión pública estadounidense como en la posterior doctrina y jurisprudencia, el caso acabará con la condena a los acusados a diez y veinte años de prisión (por incitar a la resistencia al esfuerzo bélico e instar a la reducción de la producción de material de guerra esencial) en buena muestra de que también en los Estados Unidos la información sufrirá de ciertos límites (a las que hay que sumar las relativas a la pornografía o la desestabilización del país) sin dejar de reconocer por ello su gran extensión y protección, especialmente frente al Estado.

Este paradigma liberal sufrió también de algunos cambios a lo largo del tiempo; como ocurrió durante los años ochenta del siglo $\mathrm{XX}$, cuando en pleno auge del neoliberalismo estadounidense, la Primera Enmienda fue entendida como una garantía para las empresas de comunicación frente a cualquier otro tipo de regulación gubernamental independientemente de su contenido (excepto respecto de las limitaciones por razón de la transmisión), elevando a la desinformación a la

18 "La mejor prueba para la verdad es el poder del pensamiento para ser aceptada en la competencia del mercado". Voto particular del Juez Holmes en la US Supreme Court case Abrams v. United States, 250 U.S. 616 (1919). Y continuará el juez: "Persecution for the expression of opinions seems to me perfectly logical. If you have no doubt of your premises or your power, and want a certain result with all your heart, you naturally express your wishes in law, and sweep away all opposition. To allow opposition by speech seems to indicate that you think the speech impotent, as when a man says that he has squared the circle, or that you do not care wholeheartedly for the result, or that you doubt either your power or your premises. But when men have realized that time has upset many fighting faiths, they may come to believe even more than they believe the very foundations of their own conduct that the ultimate good desired is better reached by free trade in ideas -- that the best test of truth is the power of the thought to get itself accepted in the competition of the market, and that truth is the only ground upon which their wishes safely can be carried out. That, at any rate, is the theory of our Constitution. It is an experiment, as all life is an experiment (...)." [“La persecución por la expresión de opiniones me parece perfectamente lógica. Si usted no tiene dudas sobre sus premisas o su poder, y desea un cierto resultado con todo su corazón, naturalmente expresa sus deseos en la ley y elimina toda oposición. Permitir la oposición mediante el discurso parece indicar que usted piensa que el discurso es impotente, como cuando un hombre dice que ha cuadrado el círculo, o que de todo corazón no le importa el resultado, o que duda de su poder o de sus premisas. Pero cuando los hombres se dan cuenta de que el tiempo ha trastornado muchas fes combativas, pueden llegar a creer aún más de lo que creen los fundamentos de su propia conducta, que el bien final deseado se logra mejor mediante el libre comercio de ideas: que la mejor prueba de La verdad es el poder del pensamiento para hacerse aceptar en la competencia del mercado, y esa verdad es la única base sobre la cual sus deseos pueden llevarse a cabo de manera segura. De todos modos, esa es la teoría de nuestra Constitución. Es un experimento, como toda vida es un experimento (....."] Abrams vs. United States EE.UU, 250 US 630 (1919). 
categoría de derecho. Esta postura fue criticada por Meiklejohn en 1948 en su libro Free Speech and its Relation to Self-Government argumentando que la prohibición constitucional solo estaba dirigida a su restricción, pero no a su ampliación y enriquecimiento. Por el contrario, el Congreso debía estar compelido a aumentar y mejorar la libertad de información de sus ciudadanos, promoviendo el aprendizaje, la enseñanza y un flujo informativo sin trabas, ya que - a fin de cuentas - la libertad humana no está fijada por razones meramente naturales y por lo tanto debe ser promovida ${ }^{19}$. El pensamiento de Meiklejohn, encuadrado en la mejor tradición del liberalismo, puso de manifiesto la contradicción entre un sistema mediático dirigido a "atraer a los ciudadanos a la vida pública como participantes informados" con aquel "creado simplemente para maximizar las ganancias de los inversores" ${ }^{20}$, y que tan permeable resulta a la desinformación y la actividad propagandística.

\subsection{El paradigma europeo}

En el viejo continente, la perspectiva relativa al derecho a la libertad de expresión y la libertad de prensa, aún partícipe en gran parte de la teoría liberal de los medios, será más diáfana al intervencionismo estatal. Reconocido el derecho a la información y la libertad de prensa por las constituciones europeas, que forman parte de lo que muy adecuadamente el profesor Häberle ha denominado las tradiciones constitucionales comunes (europeas), el sistema europeo se referenciará en un documento clave, que no será otro sino el Convenio Europeo para la protección de los derechos fundamentales y las libertades fundamentales, que como es conocido - establecerá el estándar mínimo a respetar. El Convenio, que se firmará tan solo dos años después de que la Declaración Universal de Derechos Humanos de 1948 establezca el derecho a la libertad de expresión y opinión en su artículo 1921, regulará también las mismas libertades.

El artículo 10.1 del Convenio establecerá pues el derecho a la libertad de expresión, de opinión y a recibir o comunicar informaciones o ideas "sin injerencia de las autoridades públicas y sin consideración de fronteras" (esta última referencia fronteriza también aparecía en la Declaración Universal ${ }^{22}$ ), pero será en su punto segundo donde se establezcan los límites a esas libertades, al señalar que "entrañan deberes y responsabilidades", y pueden ser sometidos "a ciertas formalidades, condiciones, restricciones o sanciones previstas por la ley, que constituyan medidas

\footnotetext{
${ }^{19}$ ROBERT W. MCCHESNEY, Liberalism and the media, Op. Cit.

${ }^{20}$ Ibíd.

${ }^{21}$ Declaración Universal de Derechos Humanos: “Artículo 19. Todo individuo tiene derecho a la libertad de opinión y expresión; este derecho incluye el de no ser molestado a causa de sus opiniones, el de investigar y recibir información y opiniones, y el de difundirlas, sin limitación de fronteras, por cualquier medio de expresión".

${ }^{22}$ Fórmula que también aparecerá en la redacción del artículo 19 del Pacto Internacional de Derechos Civiles y Políticos de 1976.
} 
en una sociedad democrática, para la seguridad nacional, la integridad territorial o la seguridad pública, la defensa del orden y la prevención del delito, la protección de la salud o de la moral, la protección de la reputación o de los derechos ajenos, para impedir la divulgación de informaciones confidenciales o para garantizar la autoridad y la imparcialidad del poder judicial" 23 .

Con esta redacción, que hace referencia a formalidades, condiciones, restricciones o sanciones, Europa se alejaba de una visión fielmente liberal, y daba paso a una perspectiva menos complaciente con la "mano invisible" del "mercado de las ideas". El éxito internacional de este paradigma será notable y se trasladará al artículo 19 del Pacto Internacional de Derechos Civiles y Políticos de 1976, cuyo punto 3, con una redacción más escueta, y muy en la línea de la teoría general, limitará estos derechos al aseguramiento de "el respeto a los derechos o la reputación de los demás" y "la protección de la seguridad nacional, el orden público, la salud o la moral pública" 24 .

La Unión Europea por su parte, establecerá en su Carta el derecho a la libertad de expresión e información en su artículo 11, con una redacción notablemente influida por la Convención, que introducirá una coletilla a favor de la libertad de los medios de comunicación y su pluralismo ${ }^{25}$, y que será de aplicación por parte

${ }^{23}$ Convenio Europeo para la protección de los derechos fundamentales y las libertades fundamentales: “Artículo 10. Libertad de expresión. 1. Toda persona tiene derecho a la libertad de expresión. Este derecho comprende la libertad de opinión y la libertad de recibir o de comunicar informaciones o ideas sin que pueda haber injerencia de autoridades públicas y sin consideración de fronteras. El presente artículo no impide que los Estados sometan las empresas de radiodifusión, de cinematografía o de televisión a un régimen de autorización previa. 2 . El ejercicio de estas libertades, que entrañan deberes y responsabilidades, podrá ser sometido a ciertas formalidades, condiciones, restricciones o sanciones previstas por la ley, que constituyan medidas necesarias, en una sociedad democrática, para la seguridad nacional, la integridad territorial o la seguridad pública, la defensa del orden y la prevención del delito, la protección de la salud o de la moral, la protección de la reputación o de los derechos ajenos, para impedir la divulgación de informaciones confidenciales o para garantizar la autoridad y la imparcialidad del poder judicial".

${ }^{24}$ Pacto Internacional de Derechos Civiles y Políticos de 1976: "Artículo 19. 1. Nadie podrá ser molestado a causa de sus opiniones. 2. Toda persona tiene derecho a la libertad de expresión; este derecho comprende la libertad de buscar, recibir y difundir informaciones e ideas de toda índole, sin consideración de fronteras, ya sea oralmente, por escrito o en forma impresa o artística, o por cualquier otro procedimiento de su elección. 3 . El ejercicio del derecho previsto en el párrafo 2 de este artículo entraña deberes y responsabilidades especiales. Por consiguiente, puede estar sujeto a ciertas restricciones, que deberán, sin embargo, estar expresamente fijadas por la ley y ser necesarias para: a) Asegurar el respeto a los derechos o a la reputación de los demás; b) La protección de la seguridad nacional, el orden público la salud o la moral pública".

${ }^{25}$ Carta de los derechos fundamentales de la Unión Europea: "Artículo 11 de la Libertad de expresión y de información. 1. Toda persona tiene derecho a la libertad de expresión. Este derecho comprende la libertad de opinión y la libertad de recibir o de comunicar informaciones o ideas sin que pueda haber injerencia de autoridades públicas y sin consideración de fronteras. 2. Se respetan la libertad de los medios de comunicación y su pluralismo". 
de las instituciones europeas y por la de los Estados miembros cuando apliquen Derecho de la Unión.

Cuáles son las limitaciones de la libertad de expresión del informante y especialmente si existe una libertad para desinformar será la cuestión de fondo que se agitará con la aparición de las fake news.

En primer lugar, resultará necesario descubrir el triple vínculo que une a la libertad de expresión con la libertad de acceso a la información, y el de ambas con el derecho a la participación de la ciudadanía en los asuntos públicos.

Dado que no puede haber comunicación sin recepción, podemos afirmar que el derecho a la información es en realidad una precisión del derecho a expresar el pensamiento de cada uno. Como además todos los derechos - y en particular estos - conciernen a todos, debemos concluir que existe un interés general (la dimensión institucional del derecho de expresión e información) de que la información llegue a toda la ciudadanía, transformando la libertad de información "en el resultado social del ejercicio de la libertad de expresión" 26 . Una cuestión, que en términos democráticos no puede restringirse únicamente a la autonomía personal, sino que debe "aspirar a asegurar a los receptores la posibilidad de apreciar la más diversa variedad de puntos de vista en torno a un tema determinado" 27 .

Es por ello, que la libertad de información no solo se encarga de proteger al comunicante, sino que abarca todo el proceso de elaboración, búsqueda, investigación, selección y confección de la información, es decir, toda su actividad preparatoria $^{28}$. Una labor, nada sencilla, que requiere del despliegue de una serie de competencias, habilidades y técnicas propias de aquellos que "hacen de la búsqueda y difusión de la información su profesión específica", tal y como ha señalado, por ejemplo, el Tribunal Constitucional español ${ }^{29}$. Por consiguiente, si bien el derecho a la libertad de información concierne a todos los ciudadanos, posee una especial relevancia respecto de los periodistas, que por ello detentan obligaciones específicas ${ }^{30}$. Y es aquí donde la intervención administrativa legitima su actuación, debido al papel que el derecho a la información y sus profesionales juegan en la sociedad. No puede olvidarse en este punto que, su fin último es

${ }^{26}$ CRISAFULLI, V., "Problemática della 'Libertà d'informazione'”, Il Politico, 1964, 285 y ss. En: SOLOZABAL ECHAVARRIA, J.J., "Aspectos constitucionales de la libertad de expresión y el derecho a la información", Revista Española de Derecho Constitucional, 23, 1988,142.

${ }^{27}$ SABA, R., "El derecho de la persona a acceder a la información en poder del gobierno", Derecho Comparado de la Información, $\mathrm{n}^{\mathbf{0}}$ 3, enero-junio, 2004, p.145-185. En web (20/04/2019): http://ru.iis.sociales.unam.mx/bitstream/IIS/5301/1/Leyes\%20de\%20Acceso $\% 20$ a $\% 201$ \% $\% 20$ Inform aci\%C3\%B3n\%20en\%20el\%20Mundo.pdf

${ }^{28}$ SOLOZÁBAL, J.J., "Aspectos constitucionales de la libertad de expresión y el derecho a la información", Op. Cit.

${ }^{29}$ Sentencia del Tribunal Constitucional 6/1981, de 16 de marzo de 1981.

${ }^{30}$ Véase: NAVAS, M., Derechos fundamentales de la comunicación: una visión ciudadana, Ecuador, Editorial Abya Yala, 2002. En web (12/04/2019): http://dspace.udla.edu.ec/bitstream/33000/9274/1/UDLA-EC-TAB-2018-07.pdf 
asegurar la participación de la ciudadanía en los asuntos públicos y en consecuencia evitar la apropiación indebida del espacio público que pertenece a todos (al pueblo soberano) y se respeten las reglas de juego ${ }^{31}$. Al respecto, el Tribunal de Estrasburgo ha tenido la oportunidad de precisar en Stoll v. Switzerland de 2007 que "la salvaguardia que otorga el Artículo 10 al periodista en relación con la presentación de informes sobre temas de interés general está sujeta a la condición de que actúen de buena fe y sobre una base fáctica precisa y proporcionen "información confiable y precisa de acuerdo con la ética del periodismo"'”32; una decisión jurisprudencial que por cierto no pasó inadvertida para el HLEG ${ }^{33}$.

Por otra parte, respecto de la comunicación protegida por la libertad de expresión y comunicación parece necesario distinguir dos actividades bien distintas que nos permitirán distinguir los límites a la libertad de expresión del informante, que como hemos visto no puede ser absoluta, si no se quiere correr el riesgo de desvirtuar su fin último democrático. Como el Tribunal Constitucional español ha señalado en reiterada jurisprudencia (como en la STC 34/1996) es necesario distinguir dos actividades informativas bien distintas, que no obstante vamos a encontrar entremezcladas en la realidad. Aquella expresión que refleja una opinión o una idea, de aquella que divulga una información o una noticia. En demasiadas ocasiones entremezcladas, el alto tribunal español ha resuelto su posible confusión a través de la teoría del elemento preponderante. Esta teoría estará dirigida a permitir reconocer cuando prevalece el componente ideológico o de opinión, en cuyo caso prevalece el derecho a la libertad de opinión, de cuando estemos en presencia de una noticia o actividad informativa, que se hallará amparada mediante el ejercicio de la libertad de información. El primer caso gozará - obviamente - de una libertad más amplia, mientras que el segundo (la divulgación de las noticias) exigirá veracidad ${ }^{34}$. Una técnica primordial para enfrentar el problema de las fake news, en las que la imbricación entre opinión e información va a ser especialmente estrecha, ya que como el propio HLEG señalaba, estamos en presencia de un tipo de "información fabricada mezclada con hechos y prácticas".

\footnotetext{
${ }^{31}$ MERINO, M., “Transparencia: Libros, Autores e Ideas", Instituto Federal de Acceso a la Información Pública/CIDE, México Cit, 2005, p.11-20. En web (20/04/2019): http://ru.iis.sociales.unam.mx/bitstream/IIS/5301/1/Leyes\%20de\%20Acceso $\% 20$ a $\% 201$ a $\% 20$ Inform aci\%C3\%B3n\%20en\%20el\%20Mundo.pdf

32 "The safeguard afforded by Article 10 to journalist in relation to reporting on issues of general interest is subject to the proviso that they are acting in goo faith and on an accurate factual basis and provide "reliable and precise information in accordance with the ethics of journalism" Stoll v. Switzerland [GC] NO 69698/01 ECHR 2007 -XIV.

33 EUROPEAN COMMISSION. DIRECTORATE-GENERAL FOR COMMUNICATION NETWORKS, CONTENT AND TECHNOLOGY. "A multi-dimensional approach to disinformation: Report of the independent high-level group on fake news and online disinformation", Op. Cit., 18.

${ }^{34}$ Véase: PÉREZ ROYO, J. Curso de Derecho Constitucional, 14 Edición, Marcial Pons, Sevilla, 318-319.
} 
Pero esta no será la única característica del fenómeno que pueda resultar relevante desde el punto de vista jurídico.

\section{$3 \quad$ ¿Un fenómeno online?}

\subsection{Las fake news como flujo desinformativo}

La definición del término Fake News realizada por el diccionario Cambridge señala directa y principalmente a Internet como el lugar de difusión de estas actividades, aunque matice inmediatamente dicha afirmación al señalar también a "otros medios". La cuestión es importante, pues significa circunscribir el fenómeno, al menos de forma preponderante, al ciberespacio; cuestión nada baladí, y que nos conduce a la trascendental pregunta sobre si podemos deducir de ello algún tipo de consecuencia jurídica.

Siguiendo la definición de la HLEG, la eficacia de gran parte de las actividades de confección o difusión de las desinformaciones parecen necesitar de la concurrencia de Internet e incluso de la utilización de la inteligencia artificial. Las actividades (a veces tan simples, a veces tan sofisticadas) como el astroturfing, las redes de trolling, los miembros visuales o el mero tuitear de estas noticias ${ }^{35}$ no pueden imaginarse fuera del contemporáneo entorno tecnológico, teniendo muy en cuenta que creación y difusión son el anverso y reverso, respectivamente, del derecho de expresión-información.

La relación entre tecnología e información es una relación muy antigua (como lo es también para el Derecho ${ }^{36}$ ) y sus hitos están tan íntimamente relacionados (la imprenta y la prensa escrita, la radiodifusión y la radio, la televisión, etc.) que han condicionado como las hemos entendido. El sociólogo Manuel Castells ha denominado a nuestro tiempo como la era de la información, cuyo origen se remontaría a las décadas de los sesenta y los setenta, debido precisamente a la revolución microelectrónica que tuvo lugar en los Estados Unidos en aquel momento ${ }^{37}$. En opinión de Castells, la era de la información ha conformado toda una sociedad red, interconectada en tiempo real, lo que caracteriza gran parte de sus relaciones. La red resulta un espacio de flujos que está constituido por los lugares en los que "se localizan las actividades (y las personas que las ejecutan), las redes de comunicación material que vinculan estas actividades, y el contenido y la geometría de los flujos de información (...) [generando] función y significado" 38 . La

35 EUROPEAN COMMISSION. DIRECTORATE-GENERAL FOR COMMUNICATION NETWORKS, CONTENT AND TECHNOLOGY. A multi-dimensional approach to disinformation: Report of the independent high-level group on fake news and online disinformation, Op. Cit., 10.

36 Véase: ARJONA SANCHEZ, M. J., "El Derecho en Red", Videtur Quod: anuario del pensamiento crítico 2, 2010, 1-183.

${ }^{37}$ CASTELLS, M., Comunicación y Poder, Siglo XXI Editores, México, 2013, 49.

${ }^{38}$ Ibíd. 63. 
descripción es importante porque no dibuja un espacio tal y como estamos acostumbrados a imaginarlo. En el mundo descrito por Castells, el espacio de la información no es ya el periódico o la pantalla, sino el flujo informativo que genera función y significado, y que en última instancia está constituido por la mente de los receptores, apoyados - eso sí - por la tecnología ${ }^{39}$. Desde este punto de vista no parece descabellado afirmar que la singularidad de las fake news no está constituida por su contenido (los bulos, las medias verdades, el uso de la sugestión para desinformar ya está presente en la prensa amarilla), ni por su concepción o forma de creación (pues con más o menos sofisticación ya está presente en el amarillismo) y, desde luego, tampoco en su capacidad de difusión masiva (característica de los mass media como demostraron Chomsky y Herman), sino probablemente por el espacio en el que se desarrollan, que no es exactamente Internet, sino las conexiones en red que caracterizan precisamente la sociedad red. Las fake news no son en realidad fragmentos de desinformación (unas noticias falsas) sino el flujo en el que se desarrollan, alteran y perviven. Por así decirlo, una fake news no es una noticia falsa, sino todo el flujo de desinformaciones, reacciones y comunicaciones que ese determinado fragmento de desinformación es capaz de generar. Las fake news son la trama de flujos de comunicación, transformación, retroalimentación, etc. destinados a desinformar, de ahí su dificultad de aprehensión, especialmente para el Derecho.

\subsection{La oportunidad de un régimen especial}

Ante estos flujos de desinformación, que gracias a las nuevas tecnologías incrementan de forma muy notable sus efectos, la gran pregunta que se plantea no es otra sino aquella sobre la legitimidad constitucional y la oportunidad de desarrollar un régimen especial para la manifestación del pensamiento en red, es decir, si el actual paradigma constitucional de la tutela del pluralismo informativo es aún valido en nuestro ecosistema digital de la información ${ }^{40}$. Como algún autor ha señalado partimos de una posición generosa con la libertad de información, según la cual, la difusión de una noticia falsa no puede ser considerada ilícita en sí y por sí misma, sino cuando constituya el punto de apoyo de una actividad ilícita que afecte a otros principios constitucionales ${ }^{41}$. Una cuestión que nos conduce de nuevo a los principios sobre los que hemos construido la tutela de estos derechos.

Bajo el paradigma estadounidense se hace necesario volver a recurrir a la metáfora del mercado de las ideas, e interrogarnos sobre si la red constituye en sí

\footnotetext{
${ }^{39}$ Véase: Ibíd. Castells no pretende que estos flujos sean nuevos, sino que se han hecho más evidentes que nunca gracias a la interconectividad que la tecnología ha permitido.

40 VIGEVANI, G., AND M. BASSINI. "Primi appunti su fake news e dintorni", 2017, 11-22, 13-14. En este artículo se hace referencia a la polémica desarrollada en la prensa italiana a raíz de las propuestas de regulación de la actividad informativa en la red y la "l'Autorità della Veridicità".

${ }^{41}$ BARILE, P, Diritti dell'uomo e libertà fondamentali, Bologna, 1984, 229.
} 
misma un mercado de las ideas distinto al tradicional, y si - por lo tanto - reclama una visión diferente sobre el fenómeno. El Tribunal Supremo de los Estados Unidos ha tenido la oportunidad de referirse a esta cuestión (véase Reno v. ACLU de 1997) $\mathrm{y}$ ha decidido no modificar su acercamiento tradicional a la cuestión. Por el contrario, el mercado digital de las ideas, al estar compuesto por varios guardianes (plataformas informativas, redes sociales) que actúan en un "contexto policéntrico. parece mucho más adecuado para mitigar los posibles efectos nocivos derivados de la circulación de estas noticias que el mercado tradicional, pues genera "un contexto competitivo entre las fuentes" que cada día resulta más plural. En otras palabras, "el escenario de información des-intermediada, en el que los usuarios a veces se convierten creadores-usuarios, parece mitigar los efectos negativos relacionados con la difusión de noticias falsas", al menos en comparación con las posibilidades ofrecidas por el mercado tradicional ${ }^{42}$.

De igual modo la legislación estadounidense parece remachar este enfoque. Así, la sección 230 de la Ley Federal de Decencia en las Comunicaciones de 1996 (CDA) protege a los proveedores de contenido respecto de las acciones por difamación y otros delitos relativos a la libertad de expresión, cuando la información causante de la injuria haya sido proporcionada por otro usuario de Internet ${ }^{43}$, cuya responsabilidad corresponde al autor original de la publicación difamatoria. La línea divisoria para definir si una entidad es un proveedor de servicios de Internet o un proveedor de contenido de Internet dependerá, por un lado, de la función de publicador/editor; y por otro lado, de la declaración respectiva (esto es, que haya sido hecha por el proveedor de contenido) ${ }^{44}$.

En Europa la cuestión ha sido enfrentada de forma esencialmente distinta. Alertada la sociedad europea por la capacidad de estos flujos de información de abrirse, varias han sido las estrategias puestas en marcha.

En Alemania, una nueva ley, que entró en vigor el 1 de octubre de 2017, obliga a una conducta activa de las redes sociales frente a estos contenidos. La ley se aplica a los proveedores de servicios y contenidos que cuentan con plataformas lucrativas y tiene como principal objetivo a aquellas plataformas que cuenten con más de dos millones de usuarios registrados. La nueva normativa obliga a la remoción de bloques de contenido, dentro de unos plazos muy breves, que serán de 24 horas desde la reclamación del contenido, cuando este sea "manifiestamente ilícito", y dentro del término de la segunda jornada cuando no sea manifiesto y la licitud dependa de la falsedad del contenido o de las circunstancias del hecho ${ }^{45}$. Para el

42 VIGEVANI, G., AND M. BASSINI. "Primi appunti su fake news e dintorni", Op. Cit., 17.

${ }^{43}$ Ley de Telecomunicaciones, Congreso de Estados Unidos, 1996, Título V.

${ }^{44}$ MiNC LAW BLOG,"What is Section 230 of the Communication DeCENCY ACt (CDA)?", MINCLAW. EN WEB (12/06/2019): HTTPS://WWW.MINCLAW.COM/LEGAL-RESOURCE-CENTER/WHAT-ISSECTION-230-OF-THE-COMMUNICATION-DECENCY-ACT-CDA/

${ }^{45}$ VIGEVANI, G., AND M. BASSINI. "Primi appunti su fake news e dintorni", Op. Cit. 19. 
caso de incumplimiento, las sanciones administrativas oscilan entre 500.000 hasta 50 millones de euros.

La nueva ley se ha mostrado muy eficaz modificando la actividad de estas empresas, pero con ella se plantean serias repercusiones para la libertad de expresión y el papel que se les otorga a estas empresas. Al respecto, y en relación con un caso similar, el Tribunal de Estrasburgo tuvo la oportunidad de expresarse, en concreto en el caso Delfi. AS. v. Estonia de 2013. El caso, que trataba la condena de un proveedor de servicios, al que se le consideraba un editor y no un mero intermediario respecto de una desinformación, se saldó con la decisión de que no se había dañado el derecho asegurado por el artículo 10 del Convenio y por lo tanto la sanción no violaba el derecho. En contra se manifestaron los jueces Sajo y Tsotsoria cuyas opiniones disidentes señalaron que si prosperara este tipo de soluciones se correría el riesgo de generar como efecto la incentivación de formas de censura privada por parte de los operadores de internet, generándose un medio ambiente que restringiría la libertad frente a la censura ${ }^{46}$.

\section{3 ¿Nuevas elites? Netocracy and Code}

La distinta evolución de la libertad de expresión entre el viejo y el nuevo continente bien podría estar relacionada con los muy diferentes orígenes del constitucionalismo estadounidense y europeo. Si en el viejo continente los procesos de ruptura con el antiguo régimen fueron en general muy traumáticos generando nuevas élites, el constitucionalismo estadounidense tuvo una muy particular forma de nacer.

En este sentido el contraste entre la revolución estadounidense y francesa es muy paradigmático. En las colonias americanas no existía ninguna clase social equiparable a la aristocracia francesa, ni por supuesto a los desheredados o sansculottes franceses (si excluimos a los esclavos africanos que ni siquiera fueron considerados ciudadanos y fueron apartados del momento revolucionario). De hecho, los colonos americanos disfrutaban de un grado de libertad superior a la de sus correligionarios británicos al otro lado del atlántico, por lo que parece necesario concluir que la revolución americana fue hecha más por elites que querían mantener sus poderes frente a una metrópoli que quería reafirmar los no ejercidos durante mucho tiempo, que por verdaderos rupturistas con el orden social ${ }^{47}$.

Muy imbricadas con el mantenimiento o fractura del orden social, las libertades de expresión e información debieron conformarse y evolucionar también teniendo en cuenta estas circunstancias. La propiedad de la imprenta permitía la difusión de

\footnotetext{
46 Ibíd., 20.

47 SUANZES-CARPEGNA, J. V., "Política y Constitución en la historia británica y estadounidense. Entrevista al profesor MJC Vile", Historia constitucional: Revista Electrónica de Historia Constitucional, (10), 2009, 535-558, 545-546. http://dx.doi.org/10.17811/hc.v0i10.248
} 
las nuevas ideas liberales, pero los grupos que detentaban estas propiedades fueron distintos a un lado u otro del atlántico. En las ex-colonias americanas, las elites eran esencialmente las mismas que en el periodo prerrevolucionario. Para estos grupos, la ruptura con el Imperio Británico no debió verse como un momento en el que la estabilidad social debiera estar comprometida, y por lo tanto la libertad de expresión e información, en todo momento favorable ante las nuevas élites, no debía verse estrechada ni comprometida. En una Europa, sin embargo, sometida a unas tensiones muy mayores, la libertad de imprenta nació ya sometida a un corsé más ajustado, pues dependía de equilibrios sociales mucho más delicados. Este bien pudiera ser el caso español, donde una de sus primeras referencias a la libertad de expresión, la encontramos ya unida a la censura. Así, el Decreto IX, de 10 de noviembre, promulgado por las Cortes de Cádiz en 1810 que reconoce la libertad de imprenta, ya incluía en su artículo 13 las denominadas "Juntas de Censura", con la finalidad de "asegurar la libertad de imprenta y contener al mismo tiempo su abuso" 48 . De hecho hay que recordar que la tan liberal metáfora del mercado de las ideas estadounidense mantiene precisamente su límite en el mantenimiento de ciertos límites al orden social, tal y como se impuso en la sentencia Abrams v. United States de 1919.

En este sentido cabe interrogarnos sobre si el medio digital genera unas nuevas élites, y por lo tanto puede terminar comprometiendo estos paradigmas. En el año 2000 dos filósofos escandinavos (Alexander Bard y Jan Soderqvist) publicaron una singular obra denominada "Netocracy: The new power elite and life after capitalism" ${ }^{49}$, en la que señalaban la irrupción de una nueva clase social correspondiente a la era digital que vendría determinada por la capacidad de desarrollar el flujo de informaciones que Castells ya había anticipado. La obra que adelantaba acontecimientos que han tenido lugar con posteriori respecto de la información y la desinformación señalaba que igual que la aparición de la imprenta supuso un cataclismo informativo que cambio la sociedad, Internet tendría un efecto similar conformando nuevos grupos y elites sociales hasta llegar a afirmar que los capitalistas se convertirán en una clase inferior a los capitalistas ${ }^{50}$.

¿Pero demanda este nuevo territorio del ciberespacio en manos de esta nueva Netocracia un nuevo paradigma constitucional? Y en caso de que respondamos positivamente, ¿en qué puede consistir este?

El profesor Lawrence Lessig publicó en 1999 "Code and other Laws of Ciberspace" ${ }^{\prime 51}$ en la que argumentaba que el Derecho debía comprender como la

${ }^{48}$ BIBLIOTECA VIRTUAL MIGUEL DE CERVANTES, Madrid, 2006.

${ }^{49}$ BARD, B Y JAN SODERQVIST, J, Netocracy: The new power elite and life after capitalism, Pearson FT Press, 2000.

${ }^{50}$ BARD, B Y JAN SODERQVIST, J, Netocracia: El nuevo poder en la red y la vida después del capitalismo, Prentice Hall, 2003, 213.

${ }^{51}$ LESSIG, L, Code and other law of cyberspace, 1999. 
estructura fundamental de la red viene determinada por el Código software, y que este que regula las conductas humanas como las de la sociedad protagonizada por la imprenta viene regulada por "le code". En nuestra opinión, en la propuesta de Lessig se encuentra la solución a gran parte del reto jurídico no solo a las actuales fake news sino a los retos que la inteligencia artificial nos deparará durante las siguientes décadas. Si la libertad de información no solo se encarga de proteger al comunicante, sino que abarca todo el proceso de elaboración, búsqueda, investigación, selección y confección de la información, es decir, toda su actividad preparatoria ${ }^{52}$, no podemos dejar pasar por alto que gran parte de estas actividades son llevadas a través de plataformas digitales o desarrolladas directamente a través de la inteligencia artificial, mediante redes cuyo comportamiento o actividades que le permiten al usuario viene determinado por el software, es decir, por el contenido habilitado o deshabilitado por el código informático de estas. Dada la buena fe que se les exige a estas actividades preparatorias (ya se les exige a los periodistas) se trataría de regular qué actividades no deberían permitírseles a estas plataformas o máquinas, evitando actividades que impidan o dificulten la reflexión de sus usuarios-lectores, como los flashes de noticias que desaparecen sin permitirle al lector la necesaria reflexión, los contenidos subliminales, la confección en base a datos personales recolectados, etc. Actividades todas ellas realizadas a través de flujos cuya velocidad hace muy difícil la aprensión sin recurrir a los siempre indeseados mecanismos de censura, pero que pudieran ser controlados no desde su contenido sino desde la forma que adoptan. Así sin necesidad de censurar los contenidos, deberían limitarse determinadas actividades de las que solo puede presumirse su mala fe, o al menos deben ser consideradas no suficientemente éticas. Limitando el código de lo que se puede hacer o no y de lo que se debe o se pueda hacer en cada cadena de estos flujos, serían estos mismos los que permitirían que una audiencia con mayor tiempo de reflexión, pudieran aprovechar la pluralidad que los nuevos medios representan, sin caer en las cadenas de desinformación amparadas en el anonimato, la velocidad de contenidos, la falta de reflexión y los demás peligros que representan. Si "la salvaguardia que otorga el Artículo 10 del Convenio exige que la actividad del periodista esté sujeta a la buena fe y sobre una base fáctica precisa para que proporcionen "información confiable y precisa de acuerdo con la ética del periodismo"" 53 deberían determinarse que procesos deben ser considerados indeseables en la actividad de estas plataformas y limitarlos a través de sus códigos informáticos.

52 SOLOZÁBAL, J.J., "Aspectos constitucionales de la libertad de expresión y el derecho a la información”, Revista Española de Derecho Constitucional, no 23, Madrid, 1988.

53 "The safeguard afforded by Article 10 to journalist in relation to reporting on issues of general interest is subject to the proviso that they are acting in goo faith and on an accurate factual basis and provide "reliable and precise information in accordance with the ethics of journalism" Stoll v. Switzerland [GC] NO 69698/01 ECHR 2007 -XIV. 


\section{REFERENCES}

ARJONA SANCHEZ, M. J., "El Derecho en Red", Videtur Quod: anuario del pensamiento crítico 2, 2010, 1-183.

BARD, B Y JAN SODERQVIST, J, Netocracy: The new power elite and life after capitalism, Pearson FT Press, 2000.

BARD, B Y JAN SODERQVIST, J, Netocracia: El nuevo poder en la red y la vida después del capitalismo, Prentice Hall, 2003.

BARILE, P, Diritti dell'uomo e libertà fondamentali, Bologna, 1984.

CASTELLS, M., Comunicación y Poder, Siglo XXI Editores, México, 2013

CHOMSKY, N., 10 strategies of manipulation» by the media, 2011. URL:

http://parisis. files. wordpress. com/2011/01/noam-chomsky.pdf

CRISAFULLI, V., "Problemática della 'Libertà d'informazione"”, Il Politico, 1964, 285 y ss. En: SOLOZABAL ECHAVARRIA, J.J., "Aspectos constitucionales de la libertad de expresión y el derecho a la información", Revista Española de Derecho Constitucional, 23, 1988.

EUROPEAN COMMISSION. DIRECTORATE-GENERAL FOR COMMUNICATION NETWORKS, CONTENT AND TECHNOLOGY. “A multidimensional approach to disinformation: Report of the independent high-level group on fake news and online disinformation", Publications Office of the European Union, 2018. Disponible en Internet:

https://ec.europa.eu/digital-single-market/en/news/final-report-high-level-expertgroup-fake-news-and-online-disinformation

HERMAN, E. S., \& CHOMSKY, N., Manufacturing consent: The political economy of the mass media, Random House, 2010.

KANS, M., Freedom of Speech, Yale Law School, 2009.

LESSIG, L, Code and other law of cyberspace, 1999. 
LIPPMANN, W., Public Opinion. 1922. URL: http://infomotions. com/etexts/gutenberg/dirs/etext04/pbp nn10. htm.

MERINO, M., "Transparencia: Libros, Autores e Ideas", Instituto Federal de Acceso a la Información Pública/CIDE, México Cit, 2005. En web (20/04/2019):

http://ru.iis.sociales.unam.mx/bitstream/IIS/5301/1/Leyes $\% 20 \mathrm{de} \% 20$ Acceso $\% 20 \mathrm{a} \%$ 201a\%20Informaci\%C3\%B3n\%20en\%20el\%20Mundo.pdf

MINC LAW BLOG, "What is Section 230 of the Communication Decency Act (CDA)?", MincLaw. En web (12/06/2019): https://www.minclaw.com/legal-resourcecenter/what-is-section-230-of-the-communication-decency-act-cda/

NAVAS, M., Derechos fundamentales de la comunicación: una visión ciudadana, Ecuador, Editorial Abya Yala, 2002. En web (12/04/2019):

http://dspace.udla.edu.ec/bitstream/33000/9274/1/UDLA-EC-TAB-2018-07.pdf

PÉREZ ROYO, J. Curso de Derecho Constitucional, 14 Edición, Marcial Pons, Sevilla. ROBERT W. MCCHESNEY, "Liberalism and the media. Open Democracy blog”, 2014. URL: https://www.opendemocracy.net/en/liberalism-and-media/

RODRÍGUEZ-POLO, X. R., \& ALGARRA, M. M., Los principios del sistema liberal de medios ante la crisis de la comunicación pública. Una aproximación crítica. Vivat Academia, 2011.

SABA, R., "El derecho de la persona a acceder a la información en poder del gobierno", Derecho Comparado de la Información, no 3, enero-junio, 2004, p.145-185. En web (20/04/2019): http://ru.iis.sociales.unam.mx/bitstream/IIS/5301/1/Leyes $\% 20 \mathrm{de} \% 20$ Acceso $\% 20 \mathrm{a} \%$ 20la\%20Informaci\%C3\%B3n\%20en\%20el\%20Mundo.pdf

SOLOZÁBAL, J.J., “Aspectos constitucionales de la libertad de expresión y el derecho a la información”, Revista Española de Derecho Constitucional, no 23, Madrid, 1988.

SUANZES-CARPEGNA, J. V., "Política y Constitución en la historia británica y estadounidense. Entrevista al profesor MJC Vile", Historia constitucional: Revista Electrónica de Historia Constitucional, (10), 2009, 535-558.

http://dx.doi.org/10.17811/hc.v0i10.248 
VIGEVANI, G., AND M. BASSINI, "Primi appunti su fake news e dintorni", 2017, $11-22$.

WARDLE, C Y DERAKHSHAN, H "Information disorder: Toward an interdisciplinary framework for research and policy making", Council of Europe, Strasbourg, 2017. 